\title{
Epistemologia da comunicação, neomaterialismo e cultura digital
}

\author{
André Lemos' \\ https://orcid.org/0000-0001-9291-6494 \\ I - Universidade Federal da Bahia. Salvador \\ Salvador (BA), Brasil
}

Resumo: O artigo apresenta teorias neomaterialistas, visando sua aplicação no campo da comunicação social. É proposto o conceito de mediação como central para um entendimento dos processos comunicativos que leve em consideração a agência dos objetos e sua materialidade. Parte-se da hipótese de que boa parte dos estudos de comunicação valoriza perspectivas antropocêntricas, relações intersubjetivas, contextuais e transcendentes. Essa postura não seria capaz de abarcar toda a complexidade dos fenômenos comunicacionais, em geral, e os da cultura digital, em particular. O artigo apresenta uma proposta metodológica para análise neomaterialista de objetos infocomunicacionais.

Palavras-Chave: materialidade; mediação; comunicação social; cibercultura.

Abstract: Epistemology of communication, neomaterialism and digital culture - This paper aims to present neomaterialist theories, aiming its application in the social communication domain. The concept of mediation is proposed as central to understand the communicative process, taking seriously the agency of objects and their materiality. The article starts from the hypothesis that the majority of communication studies values anthropocentric perspectives, intersubjective, contextual and transcendent relations. This posture would not be able to encompass all the complexity of communicational phenomena in general, and those of digital culture in particular. The article presents a methodological proposal for a neomaterialistic analysis of infocommunicational objects.

Keywords: materiality; mediation; social communication; cyberculture.

\section{Introdução}

Esse artigo aponta as principais características das correntes neomaterialistas e propõe uma metodologia de análise de processos, produtos e objetos da cultura digital ${ }^{1}$.

1 Esse artigo faz parte da pesquisa Comunicação, Mediação e Cibercultura (PQ-1A CNPq). Essas ideias foram apresentadas no III Seminário Internacional sobre midiatização e processos sociais na UNISINOS, São Leopoldo - RS, em maio de 2019. Devo muito às discussões no interior do Lab404 (http://lab404.ufba.br). 
Na primeira parte apresentamos as principais características dessas teorias e discutimos o conceito de mediação radical. Em seguida, retomamos propostas anteriores de comunicação associal e modo de existência da comunicação (LEMOS, 2019). Na última parte são descritos os pressupostos, as etapas e os instrumentos para a aplicação de uma metodologia neomaterialista da comunicação em produtos da cultura digital.

\section{Neomaterialismo, midiatização e mediação radical}

Muitos autores apontam para uma virada materialista (material turn) (BENNETT; JOYCE, 2010), ou uma virada não humana (non-human turn) (GRUSIN, 2015a) na filosofia e nas ciências sociais, ressaltando a agência dos objetos e, consequentemente, suas performances como mediadores importantes na formação social. No entanto, essa virada parece ainda não ter influenciado o campo da comunicação social. Grande parte dos estudos de comunicação valoriza relações intersubjetivas, contextuais e transcendentes, pouco afeitas ao reconhecimento da agência dos objetos. Isso prejudicaria a análise dos fenômenos comunicacionais como um todo, e os da cultura digital em particular². Essa é a hipótese central desse artigo. Estudos de materialidade foram sempre periféricos nos estudos de comunicação (QUANDT; VON PAPE, 2010, p. 330). Lievrouw aponta acertadamente para o problema de uma forma global:

\section{[...]a maioria das bolsas de tecnologia no campo da comunicação, informada por fluxos clássicos de pesquisa em mídia, continua a seguir uma linha culturalista amplamente construtivista, privilegiando os significados e apropriações sociais e culturais das tecnologias, enquadrando a tecnologia principalmente como resultado ou expressão da cultura. Como resultado, a mudança para entender conceitualmente o caráter social e material intrínseco da tecnologia da comunicação como igualmente definitivo e codeterminante permanece como um projeto inacabado na pesquisa em comunicação e mídia (LIEVROUW, 2014, p. 24).}

Por neomaterialismo entende-se um conjunto de pressupostos surgidos nos anos 1980 e 1990, a partir de diferentes autores e escolas: teoria das materialidades de origem germânica (GUMBRECHT; PFEIFFER, 1994; KITTLER, 1999), sociologias monadológicas (BENSUSAN; FREITAS, 2018), como a Teoria Ator-Rede (LAW, 1992; LATOUR, 2005, 2015; CALLON, 2006); filosofias orientadas a objetos e agenciamentos (DE LANDA, 2005; VERBEEK, 2005; HARMAN, 2011; BENNETT, 2010), pós-humanismo, feminismo e estudos de gênero (HARAWAY, 1987; BARAD, 2007; BRAIDOTTI, 2013), entre outros. Em relação à atual cultura digital destacam-se os trabalhos de Pink et al. (2016), Lupton (2016) e Dourish $(2016,2017)$. O neomaterialismo herda argumentos desenvolvidos nos séculos XVIII, XIX e XX, tais como os de empirismo radical (HUME, 2003, JAMES, 1912),

2 Estudo preliminar em andamento no Lab404 analisa textos dos GTs "Comunicação e Epistemologia" e "Comunicação e Cibercultura" (2013-2019) da COMPÓS e indica que a perspectiva antropocêntrica é hegemônica. 
modos de existência (SAURIAU, 2009), agenciamento (DELEUZE; GUATTARI, 1980), coletivos/associação/monadologia (LEIBNIZ, 2004; TARDE, 2007), semiologia material (FOUCAULT, 2000) e realismo (WHITEHEAD, 1978).

A partir de sínteses realizada por alguns autores (BENNETT; JOYCE, 2010; BENNETT, 2010; MILLER, 2005; FOX; ALLDRED; 2017; THRIFT, 2005; GAMBLE; HANAN; NAIL, 2019), podemos dizer que as teorias neomaterialistas partem de quatro perspectivas centrais: materialismo, pragmatismo, não antropocentrismo e associativismo. Por materialismo entende-se que todo fenômeno se desenvolve em redes, produzindo efeitos ou afetações materiais. A visão não-essencialista/pragmática sustenta que o objeto (humanos e não humanos) é o que ele faz e não pode ser definido por substância, ou categorias a priori. A posição não antropocêntrica defende que a agência está distribuída na rede/ agenciamento e que o controle e a fonte da ação não são privilégios do ator humano. Tudo se dá em uma associação localizada ou conectada localmente. A abordagem associativa/ local afirma que tudo se dá em uma rede plana, sendo que as análises das controvérsias não devem partir de explicações ad hoc. Valorizam-se os processos materiais e os fluxos de agências em experiências nas quais as questões sociais são sempre resultado de coletivos humanos e não humanos ${ }^{3}$. Parte-se de uma ontologia plana, buscando sempre escapar, a priori, de análises estruturais (GIDDENS, 1999) ou interacionistas (GOFFMAN, 1975).

A chave para o entendimento desses quatro princípios está nos conceitos de empirismo radical (HUME, 2003; JAMES, 1912), mediação (LATOUR, 2005; CALLON, 2006; LAW, 1992), dispositivo (FOUCAULT, 2000), situação sintética (KNORR-CETINA, 2013), agenciamento (DELEUZE, GUATTARI, 1980; DE LANDA, 2005) e realismo agencial (BARAD, 2007). Para agrupar esses termos sob uma única denominação, vou adotar o termo mediação radical, proposto por Richard Grusin a partir da ideia de radical empiricism de W. James. Como explica o autor:

Argumento que, embora a mídia e as tecnologias da mídia tenham operado e continuem operando epistemologicamente como modos de produção de conhecimento, elas também funcionam técnica, corporal e materialmente para gerar e modular afetos individuais e coletivos ou estruturas de sentimento nos agenciamentos de humanos e não-humanos. (GRUSIN, 2015b, p. 125).

O adjetivo radical permite falar de mediação envolvendo humanos e não humanos colocando, portanto, a dimensão híbrida e materialista no seu entendimento. Todo processo comunicacional é resultado dessa mediação radical, entendida aqui como associativa, não essencialista/pragmática, material e não antropocêntrica, nos afastando do pensamento da mediação como emergente apenas de relações intersubjetivas. O conceito de mediação radical vai além da ideia de mediação como proposta pelos estudos de midiatização desenvolvidos por Couldry (2008), Hepp (2008), Hjarvard (2014, 2015), entre outros.

3 Para uma crítica da perspectiva neomaterialista ver Rekret (2018). 
Abordei em outro trabalho (LEMOS, 2019) a ideia de mediação, indicando que para as sociologias pragmáticas ela absorve ações de humanos e não humanos, valorizando a materialidade dos objetos envolvidos em processos sociocomunicacionais. Chamando agora essa mediação de radical, podemos dizer que ela é o princípio dos processos de comunicação: ruptura de isolamento, trocas semióticas em entrelaçamento, "ser-enquantoum-outro" (LATOUR, 2012), incluindo os objetos na equação. Naquela ocasião afirmava que precisaríamos avançar e pensar a comunicação como um processo associal, fruto dessa mediação radical, entendendo o social para além das relações interpessoais, indo além das trocas "entre consciências humanas" (MARTINO, 2001). O social é fruto das associações nesse coletivo de humanos e não humanos. A comunicação é assim um processo associal, fruto da mediação radical. Com essa ênfase, podemos pensar de forma mais completa e integrada como os humanos são agidos e agem no interior dos processos associativos sem retirar da equação agentes fundamentais para compreensão desses processos (os objetos e suas materialidades) (LATOUR, 2015). A compreensão da ação, da mediação e da comunicação se dá, nessa perspectiva, a partir de análises imanentes, descrevendo a ação de todos os actantes envolvidos na mediação (radical) em uma análise plana, sem recorrer a priori a explicações contextuais ou globais por um lado, ou microssociais e antropocêntricas por outro.

Retornando então às diferenças em relação à midiatização, podemos dizer que a visão neomaterialista defende uma postura epistemológica oposta. Midiatização é entendida como um processo estrutural de influência das mídias (cultura de massa e controle da comunicação). Couldry e Hepp (2017) identificam como midiatização profunda a ação das novas mídias digitais. Os estudos de mediação se dedicam ao "impacto da mídia em situações comunicativas específicas situadas no tempo e espaço" (HJARVARD, 2015, p. 53) e os de midiatização a compreender "as transformações estruturais de longo prazo no papel da mídia na sociedade e na cultura contemporâneas" (HJARVARD, 2015, p. 53). Para Hjarvard, mediação refere-se a "situações comunicativas específicas" e midiatização a "transformações estruturais na sociedade":

Discussões internacionais recentes apontam para a resolução destas discordâncias terminológicas em favor da distinção europeia continental entre "midiatização" (denotando a dimensão estrutural de longo prazo) e "mediação" (significando o uso da mídia em encontros comunicativos)' [...]. A mídia co-estrutura a comunicação e a interação (isto é, o nível da mediação), mas a midiatização ocorre através da institucionalização de padrões de interação particulares (regras formais e informais) e alocação dos recursos interacionais no interior de uma instituição social ou esfera cultural em particular. (HJARVARD, 2015, p. 53-54).

Certamente, o que os autores identificam como "importância transformadora da mídia" ou "estrutura do modus operandi das mídias" é resultado provisório e sempre 
a ser questionado de diferentes formas da mediação radical. Couldry e Hepp (2016) e Pink (2015) apontam para a necessidade de uma perspectiva não-representacional, ou seja, não centrada na mídia (non-media-centric), reconhecendo a necessidade de enfatizar aspectos materiais e sensórios das práticas midiáticas. No entanto, os estudos não são efetivamente materialistas, caracterizando o que apontaremos mais adiante como objectwashing. Muitos estudos reduzem, como afirma Finnemann (2013, p. 298), a discussão material à questão da tecnologia. Outros propõem aliar midiatização a uma perspectiva mais materialista. Knorr-Cetina (2013, p. 41-41; 49) sustenta, na análise sobre as scopic media dos operadores do mercado financeiro, uma posição sintética, atenta a como as ações enraízam-se nas práticas, nos espaços e nos objetos, indo além (melhor seria dizer, aquém) das infraestruturas.

Processos comunicacionais midiáticos levam certamente a processos estruturantes (midiatização). O interessante, na perspectiva neomaterialista, é chegar neles aos poucos, desenvolvendo uma análise local e imanente que aponte os agenciamentos em ação. No caso da cultura digital, analisar fenômenos tais como a sociabilidade em redes sociais, as fake news, a prática do selfie, a questão do design e da privacidade na internet das coisas (Internet of Things, IOT), a cultura de plataformas, partindo de uma análise macrossocial da estrutura econômica do capitalismo de dados, ou pelo viés da comunicação entre humanos em situação específica, ofereceria pouca clareza sobre os desafios comunicacionais atuais, pois não levaria em conta as materialidades e a agência dos objetos aí envolvidos (interfaces, lógica algorítmica, construção de banco de dados, princípios escondidos em documentos técnicos e patentes etc.). Quem posta quando postamos no Facebook? Pensar, nesse caso, em uma agência humana independente e soberana é investir em erro grosseiro.

A perspectiva neomaterialista aplicada aos estudos da comunicação digital vai se perguntar como algoritmos, interfaces, dispositivos, leis, regulações, patentes, redes de comunicação, espaços de uso etc. constroem determinado fenômeno. Isso evita que deixemos esses elementos de lado em discursos que parecem dizer que reconhecem os híbridos, a técnica, a mídia, mas que de fato não dedicam tempo e atenção para descrever e analisar como esses objetos afetam os humanos e as relações daí advindas. Perde-se nesse caso a visão do entrelaçamento, reduzindo o fenômeno ao contexto, à interpretação ou à estrutura por cima ou por baixo do humano - as "mediações e midiatizações". Se Barbero (1997) afirmava que deveríamos ir "dos meios às mediações", talvez agora devamos tomar a direção contrária.

Portanto, posturas antropocêntricas, contextuais ou focadas em infraestruturas, por um lado, ou micro interações (entre humanos) por outro, não ajudam a entender as principais controvérsias emergentes como os novos objetos infocomunicacionais. Deve-se descrever e analisar a rede formada na controvérsia para identificar as formas de agenciamento dos elementos em jogo, destacando suas características materiais, não essencialistas, locais. O algoritmo das plataformas digitais contemporâneas não é 
caixa-preta pois é possível entender não o que ele é (em essência), mas pragmaticamente sua agência, o que ele faz-fazer (BUCHER, 2018). Mapear, identificar e analisar as ações nessa comunicação associal é exercer um posicionamento mais míope, menos preocupado em generalizações culturais ou sociais. Uma abordagem neomaterialista permite escapar à dicotomia estabelecida pela comunicação social em direção a uma comunicação associal, reconhecendo a particularidade da mediação radical.

Na perspectiva material e pragmática, interessa saber que tipos de interface, leis, espaços, instituições, instrumentos, patentes, documentos técnicos, agência algorítmica, fichas e outros elementos materiais em entrelaçamento produzem o problema a ser investigado. Devemos nos perguntar como a rede é montada e como ela age, como elementos materiais entram em mediação e como o resultado se faz na produção desse entrelaçamento, identificando afetações imanentes, focando localmente o problema, sem apelo a noções transcendentes, pagando o preço das conexões, reconhecendo poderes e relações de força no interior da experiência (LEMOS, 2018). Poder e desigualdades se produzem no interior das redes/assemblages, eles tendem a se encapsular, mas não podemos congelar ou transferir uma análise para a outra, sob pena de perder a mediação radical localizada e em movimento, que reconhecemos como essas estabilizações.

Ronaldo Lemos (2019) escreveu um artigo na Folha de São Paulo afirmando que não há opinião pública nas redes sociais por causa da ação de bots, algoritmos, interfaces. Parte-se do princípio de que o público e sua opinião não seriam produzidos e sim desvelados por instrumentos neutros. Marres (2012), por exemplo, vai mostrar a partir do conceito de material participation o papel dos objetos nas formas de mobilização e engajamento político. O mesmo na produção do público (LIPPMANN, 2007) ou sua opinião (TARDE, 2007). Para uma perspectiva neomaterialista, a questão é como artefatos fazem emergir (produzem) o público e sua opinião, como eles aparecem agora nesse sistema sociotécnico algorítmico (diferente de sua produção pela ágora, piazza, imprensa, rádio, TV etc.). Mais interessante do que saber o que é o público e sua opinião (em essência), é ver em que condições de agenciamento eles são construídos, discutidos, disseminados. Essa é a perspectiva não essencialista, pragmática, imanente, associativa e não antropocêntrica.

\section{Metodologia neomaterialista da comunicação associal}

Propomos a seguir uma metodologia neomaterialista para ser aplicada aos produtos e processos da comunicação em geral, e da cultura digital em particular. Esse método sustenta-se na perspectiva epistemológica apresentada acima, insistindo em apontar as mediações radicais nos processos da comunicação associal. O pressuposto é que devemos reconhecer que nenhum elemento possui atributos essenciais e que eles só se definem em suas relações, nos agenciamentos, nas linhas de fuga, nos afetos, 
nas mediações radicais, portanto. É necessário partir de análises imanentes de documentos, práticas concretas, formas do discurso, lugares para identificar e descrever os elementos em mediação e as formas de afetação (transdução) na constituição da controvérsia. Os humanos não estão em um lugar privilegiado na circulação da ação, devendo dar importância aos elementos materiais e aos objetos em questão.

Em síntese, a metodologia parte dos pressupostos materialista - reconhece-se que tudo tem efeito material e existe por agenciamentos materiais - e não essencialista (pragmático) - as coisas não têm essências e são o que fazem e fazem-fazer. Esses pressupostos alinham-se a um empirismo radical para localizar afetações nos problemas identificados. Tudo é definido nos processos e nos movimentos materiais em que cada novo problema convoca novas relações de força/poder associativas: tudo o que existe, existe em relação, precisa passar por outros para continuar seu caminho de subsistência. A associação é a mediação radical - tradução, transdução, agenciamento, entrelaçamento etc. envolvendo humanos e não humanos. Sem mediação não há nada. Ela deve ser observada em uma topologia plana, local e não antropocêntrica - análises devem ser feitas levando em conta múltiplos agentes, sob pena de purificar as relações e empobrecer as descrições dos fenômenos e a identificação dos fluxos de ação. Humanos nem sempre têm a primazia da ação. Busca-se aqui ir além de perspectivas culturalistas, contextuais, hermenêuticas.

Sobre esse último princípio, identifiquei quatro tipos-ideais que aparecem, de modo geral, em pesquisas, textos e palestras de viés antropocêntrico ${ }^{4}$ sobre comunicação ou mídia digital (Maia, Object Washing, Panopticum e Ouroboros), que descrevo a seguir.

- Maia: Textos que saltam para generalizações, sem apelo à empiria. O mundo material é uma ilusão. Deve-se evitar visões centradas na concretude dos fenômenos do mundo real. Não há mundo real, a não ser o dos conceitos.

- Object-Washing: Textos que dizem reconhecer a agência dos objetos, mas não o fazem com detalhamento. Dizem identificar agências, mas saltam rapidamente para contextos. Podem reconhecer e afirmar o uso do WhatsApp para difundir fake news, mas não há análise sobre como a materialidade do WhatsApp, da rede, dos celulares, produzem esse fenômeno. O objeto é assim. A expressão é similar a eco-washing, ethical-washing (KITCHIN, 2019), privacy-washing ${ }^{5}$, designando as posições de empresas que parecem considerar os problemas correlatos (ambiente, ética, privacidade) mas que, de fato, não os levam a sério. Os estudos que fazem object-washing negam ser antropocêntricos, dizem reconhecer

4 Pesquisas em andamento estão aplicando esses "ideais-tipo" para auxiliar nas discussões da hipótese aqui levantada: a minha pesquisa "Comunicação, Mediação e Cibercultura"(PQ/CNPq) e a do Professor Elias Bitencourt (Comunicação, performatividade algorítmica e plataformas digitais. Investigações neomaterialistas acerca das experiências comunicacionais mediadas por algoritmos na era do capitalismo de dados), a ser desenvolvida no POSCOM/Lab404. A indicação desses ideais-tipo visa auxiliar na visualização do argumento geral do texto e não pretende ser uma forma de depreciação dos autores envolvidos.

5 Essa expressão está sendo usada em pesquisa sobre privacidade projetada e interfaces maliciosas (dark patterns) em desenvolvimento no Lab404. 
a formação de híbridos, os dispositivos (como Foucault), as tecnologias, mas centramse no sujeito, na estrutura, na hermenêutica, nos contextos social e cultural, no ambiente midiático.

- Panopticum: Textos que veem tudo com os mesmos olhos, presos nas clássicas teorias da comunicação, centrados nos autores canônicos, enquadrando todos os objetos em frames pré-estabelecidos e inegociáveis. Tudo é explicado sem necessidade de reconhecer a agência dos objetos ou as materialidades envolvidas. As teorias pré-estabelecidas servem para analisar qualquer estudo empírico, independentes das controvérsias em questão (agregam os tipos Maia e Object-Washing).

- Ouroboros: Os textos de grande transcendência, que parecem começar pelas grandes conclusões sobre o mundo, o universo e tudo mais. Sem qualquer empiria ou agência de não humanos, parecem que estão iniciando um debate, mas o que fazem é rodar sobre os seus próprios eixos, mordendo o próprio rabo, girando em torno de temas e assuntos, saltando da dimensão micro para a macro e vice-versa (reforçando os tipos Maia, Object-Washing e Panopticum).

Uma metodologia que vise tratar o objeto pelo pressuposto neomaterialista deve buscar reconhecer, em uma determinada controvérsia, o fluxo das mediações radicais (ou seja, a agência de humanos e não humanos sem centralidade a priori) produzindo resultados localizados e provisórios. Os modos de existência são importantes para qualificar a mediação, apontando as diferenças nos agenciamentos, as afetações e a preposição (lugar específico da mediação) a fim de produzir o falar bem, as condições de felicidade. Pode-se analisar o problema das redes sociais pelo viés de gênero, da privacidade, da vigilância, das relações intersubjetivas etc. Para cada modo, mediadores importantes aparecerão. Ele é importante como etapa de fechamento da caixa preta, reagregação do problema e indicação de proposições sobre a controvérsia em questão. Esse resultado será adicionado à própria controvérsia, servindo como um elemento na disputa sobre o problema.

Esses são os passos propostos: 1. identificação do modo, da preposição, da controvérsia; 2. identificar todos os atores envolvidos e como eles se expressam (interfaces, documentos, patentes, formas de ação etc.); 3. mapear as formas de mediação ou transdução, entendendo que ações, sentidos e forças são produzidos; 4. reagregar o problema criando mais um elemento que possa ser instituído na construção da verdade. Os instrumentos para a execução desses passos podem ser os mais variados: coleta de dados (Survey); raspagem de dados (R); codificação focada (Atlas.ti); etnografia; entrevistas; análise de documentos (patentes, fluxogramas, contratos, termos de uso etc.); grupo focal; análise de interfaces; revisão bibliográfica etc. As controvérsias podem pedir outros métodos ou mesmo a produção de ferramentas específicas. Em termos pontuais podemos sintetizar essas quatro etapas: 
- Modos: Onde se insere a questão de interesse, a preposição para produzir um bom discurso e as condições de felicidade da análise. Busca identificar em que campo de tensões o problema está indo além de uma visão contextual, construtivista ou hermenêutica;

- Inventário: Descrição da rede de objetos (humanos e não humanos) em causa na questão a ser discutida;

- Transdução: Descrição das mediações em jogo nos agenciamentos para a produção do fenômeno. Visa a abertura de caixas-pretas na identificação do que as coisas fazem, são feitas e fazem fazer;

- Reagregação: Voltar ao encaixapretamento, o resultado provisório (que será um elemento material concreto de nova mediação produzido sobre o objeto pela própria pesquisa). Dimensão propositiva com vistas a entender a afetação coletiva (humanos e não humanos), na reagregação e redefinição do problema.

\section{Conclusão}

Apontamos nesse trabalho a necessidade de levar a sério a materialidade dos processos comunicacionais, em particular os que envolvem a cultura digital, embora não seja exclusividade desse campo. As teorias neomaterialistas se sustentam a partir de quatro pressupostos: materialismo, não essencialismo, associativismo e não antropocentrismo. Indicando que tudo tem base material, tais teorias preveem que as coisas são o que fazem, que tudo está entrelaçado e que o humano não é sempre o centro das ações. O artigo sustenta que o campo da comunicação, majoritariamente antropocêntrico, pode ganhar se olhar com mais atenção para a agência material nesses processos.

Diferenciamos a ideia de mediação radical das de mediação e midiatização, mais frequentes na área de comunicação, chamando a atenção para o reconhecimento de perspectivas não antropocêntricas para pensar os seres da comunicação. Apontamos tiposideais para reconhecimento de textos e pesquisas antropocêntricas (Maia, Object-washing, Panopticum e Ouroboros). Terminamos o artigo propondo uma metodologia para os que desejam realizar estudos neomaterialistas a partir de instruções quanto aos procedimentos. Os estudos devem estar atentos à realização de um inventário, à identificação de mediação com os processos de transdução, ao modo em que o problema se articula e à reagregação como forma de proposição conclusiva sobre a controvérsia.

Os trabalhos em desenvolvimento no Lab4046 tentam aplicar essa metodologia. Estudos sobre como o Instagram afeta e é afetado por práticas profissionais; como o processo fotográfico das selfies está imbricado em práticas materiais específicas; sobre

6 Ver as pesquisas em andamento (http://www.lab404.ufba.br/?page_id=3521) e artigos publicados (http://www. lab404.ufba.br/?page_id=11). 
as fake news e a materialidade das plataformas de disseminação; o entendimento sobre a privacidade em meio à $\mathrm{PDPA}^{7}$, tendo como discussão os processos materiais de sua constituição; o uso de apropriações materiais e algorítmicas para uso da loT; as questões de gênero em aplicativos de transporte; a materialidade dos dispositivos vestíveis na configuração de um smart body; a discussão material sobre Data Comics, questionando seu status e utilizações etc. A metodologia precisa ser testada e esperamos que esse texto possa contribuir para ampliar os debates sobre as viradas materiais e não humanas na área de estudos de comunicação no país.

André Lemos é professor titular da Facom/UFBA, pesquisador $1 \mathrm{~A}$ do $\mathrm{CNPq}$ e diretor do Laboratório de Pesquisa em Mídia Digital, Redes e Espaço (Lab404), do Programa de Pós-Graduação em Comunicação e Cultura Contemporâneas (Póscom/UFBA).

almlemos@gmail.com

\section{Referências}

BARAD, K. Meeting the universe halfway: quantum physics and the entanglement of matter and meaning. Durham, Londres: Duke University Press, 2007.

MARTÍN-BARBERO, J. Dos meios às mediações: comunicação, cultura e hegemonia. Rio de Janeiro: Editora UFRJ, 1997.

BRAIDOTTI, R. The Posthuman. Cambridge: Polity Press, 2013.

BENNETT, J. Vibrant Matter: A Political Ecology of Things. Durham, Londres: Duke University Press, 2010.

BENNETT, T.; JOYCE, P. Material powers: introduction. In: (Ed.). Material Powers: Cultural Studies, History and the Material Turn. Londres: Routledge, 2010.

BENSUSAN, H.; FREITAS, J. A. A diáspora da agência. Ensaio sobre o horizonte das monadologias. Salvador: EDUFBA, 2018.

BUCHER, T. If... Then. Algorithmic power and politics. Oxford: Oxford University Press, 2018.

CALLON, M. Sociologie de I'acteur réseau. In: AKRICH, M.; CALLON, M.; LATOUR, B. (Org.). Sociologie de la Traduction. Paris: Presse des Mines, 2006, p. 267-276.

COULDRY, N. Mediatization or mediation? Alternative understandings of the emergent space of digital storytelling. New Media \& Society, v. 10, n. 3, 1 jun. 2008, p. 373-391.

; HEPP, A. The Mediated Construction of Reality. Cambridge: Polity Press, 2016.

DE LANDA, M. A New Philosophy of Society: Assemblage Theory and Social Complexity. Nova York: Continuum, 2006.

7 PDPA, acrônimo para "Plataformização, Dataficação e Performatividade Algorítmica", características centrais da cultura digital hoje. Ver LEMOS (2019a). 
DELEUZE, G.; GUATTARI, F. Mil Platôs: capitalismo e esquizofrenia, v. 1. São Paulo: Editora 34, 1995.

DOURISH, P. Algorithms and their others: algorithmic culture in context. Big Data \& Society, v. 3, n. 2, 2016, p. 1-11. Press, 2017.

The Stuff of Bits: an Essay on the Materialities of Information [Kindle Edition]. [s.I.] MIT

FINNEMANN, N. O. Digitization: New trajectories of mediatization? In: LUNDBY, K. (Ed.). Mediatization of Communication. Handbooks of Communication Science, vol 21. Berlim: de Gruyter, 2013, p. 297-321.

FOUCAULT, M. Microfísica do Poder. Rio de Janeiro: Graal, 2000.

FOX, N. J.; ALLDRED, P. Sociology and the New Materialism. Theory, Research, Action. Londres: SAGE Publications, 2017.

GAMBLE, C. N.; HANAN, J. S.; NAIL, T. What is new materialism? In: Angelaki. Journal of the Theoretical Humanities, v. 24, n.6, p. 111-134. DOI: 10.1080/0969725X.2019.1684704

GIDDENS, A.; TURNER, J. (org.). Teoria social hoje. São Paulo: Editora UNESP, 1999.

GOFFMAN, E. A representação do eu na vida cotidiana. Petrópolis: Vozes, 1975.

GRUSIN, R. (Ed.), The Nonhuman Turn. Minneapolis: University of Minnesota Press, 2015a.

p. 124-148.

Radical Mediation. Critical Inquiry, Chicago, University of Chicago Press, v. 42, n. 1, 2015b,

GUMBRECHT, H. U; PFEIFFER, K. L. Materialities of Communication. Stanford: Stanford University Press, 1994.

HARAWAY, D. A manifesto for cyborgs: Science, technology, and socialist feminism in the 1980s. Australian Feminist Studies, v. 2, n. 4, p. 1-42, 1987.

HARMAN, G. The quadruple object. Hants: Zero Books, 2011.

HJARVARD, S. Da Mediação à Midiatização: a institucionalização das novas mídias. Parágrafo, v. 2, n. 3, jul./dez, 2015, p. 51-62.

Midiatização: conceituando a mudança social e cultural. Matrizes, São Paulo, v. 8, n. 1, jan./jun, 2014, p. 21-44.

HUME, D. Investigações sobre o entendimento humano e sobre os princípios da moral. São Paulo: Editora Unesp, 2003.

JAMES, W. Essays in Radical Empiricism. Nova York, Londres: Longmans, Green, and Co, 1912.

KANNENGIEßER, S. Engaging with and reflecting on the materiality of digital media technologies: Repair and fair production. New Media \& Society, 2019. DOI: 10.177/146144819858081.

KITCHIN, R. The ethics of Smart City. RTE, 27 abr. 2019. Disponível em: https://www.rte.ie/ brainstorm/2019/0425/1045602-the-ethics-of-smart-cities/. Acesso em: 5 mai. 2019.

KITTLER, F. A. Gramophone, Film, Typewriter. Stanford: Stanford University Press, 1999.

KNORR-CETINA, K. Scopic media and global coordination: the mediatization of face-to-face encounters. In: LUNDBY, K. (Ed.). Mediatization of Communication. Handbooks of Communication Science, vol 21. Berlim: de Gruyter, 2013, p. 39-62.

LATOUR, B. Reassembling the social: an introduction to actor-network-theory. Oxford: Oxford University Press, 2005. 
2012.

Enquête sur les modes d'existence. Une anthropologie des modernes. Paris: La Découverte,

. Uma Sociologia Sem Objeto? Revista Valise, v. 5, n. 10, 2015, p. 165-187.

LAW, J. Notes on the theory of the actor-network: Ordering, strategy, and heterogeneity. Systems practice, v. 5, n. 4, p. 379-393, 1992.

LEIBNIZ, G. W. Discurso de metafísica e outros textos. São Paulo: Martins Fontes, 2004.

LEMOS, A. Comunicação e Mediação. In: ALZAMORA, G; COUTINHO, F.; ZILLER, J. Dossiê Bruno Latour. Belo Horizonte: Editora UFMG. 2019.

. Desafios Atuais da Cibercultura. In: Jornal Correio do Povo, Caderno de Sábado, Porto Alegre, 15/6/19, 2019a.

Cyberculture and Globalization. In: Oxford Research Encyclopedia of Communication. Oxford University Press. 2018.

LEMOS, R. Redes sociais não são opinião pública. Folha de São Paulo, São Paulo, 4 mar. 2019. Disponível em: https://www1.folha.uol.com.br/colunas/ronaldolemos/2019/03/redes-sociais-naosao-opiniao-publica.shtml. Acesso em: 28 set. 2019.

LIEVROUW, L.A. Materiality and Media in Communication and Technology Studies: An Unfinished Project. In: GILLESPIE, T.; BOCZKOWSKI, P.J.; FOOT, K. A. (Ed.). Media Technologies. Essays on Communication, Materiality, and Society. Cambridge: MIT Press, 2014, p. 21-51.

LIPPMANN, W. The Phantom Public. New Jersey: Transaction Publishers, 2007.

LUPTON, D. The Quantified Self. Malden: Polity Press, 2016.

MARTINO, L. C. De qual comunicação estamos falando? In: HOHLFELDT, A.; MARTINO, L.C.; FRANÇA, V. (Org.). Teorias da comunicação: conceitos, escolas e tendências. Petrópolis: Vozes, 2001, p. 11-25.

MARRES, N. Material Participation: Technology, Environment and Everyday Publics. Basingstoke: Palgrave Macmillan. 2012.

MILLER, D. Materiality. Durham, Londres: Duke University Press, 2005.

PINK, S. Approaching media through the senses: between experience and representation. Media International Australia, 154(1), 2015, p. 5-14.

; ARDÈVOL, E.; LANZENI, D. Digital materialities: design and anthropology. [s.l.] Bloomsbury Publishing, 2016.

QUANDT, T; VON PAPE, T. Living in the mediatope: a multimethod study on the evolution of media technologies in the domestic environment. The Information Society, 26(5), p. 330-345, 2010.

REKRET, P. The head, the hand, and the matter: new materialism and the politics of knowledge. In: Theory, Culture and Society, O(0), 2018, p. 1-24. DOI: 10.1177/0263276418806369.

SOURIAU, E. Les différents modes d'existence. Paris: PUF, 2009.

STERNE, J. "What Do We Want?" "Materiality!" "When Do We Want It?" "Now!". In: GILLESPIE, T.; BOCZKOWSKI, P.J.; FOOT, K. A. (Ed.). Media Technologies. Essays on Communication, Materiality, and Society. Cambridge: MIT Press. 2014, p.119-128.

TARDE, G. Monadologia e sociologia - e outros ensaios. São Paulo: Cosac Naify, 2007.

THRIFT, N. Beyond mediation: three new material registers and their consequences. In: MILLER, D. (ed.). Materiality. Durham, Londres: Duke University Press, 2005, p. 231-256. 
VERBEEK, P. What Things Do. Philosophical Reflections On Technology, Agency, and Design, v. 1. Pensilvânia: Pennsylvania State University Press, 2005.

WHITEHEAD, A. N. Process and Reality, corrected edition. Nova York: Free Press, 1978.

Artigo recebido em 11/07/2019

e aprovado em 27/09/2019 